\title{
Como o ginecologista pode orientar mulheres com antecedentes familiares de câncer?
}

\author{
How the gynecologist can guide women with a family \\ bistory of cancer?
}

Os cânceres ginecológicos representam uma parcela considerável da morbidade e mortalidade por neoplasia. Segundo o INCA, cânceres como o câncer de mama acometerão, em 2014, 57.120 mulheres, o câncer de ovário, 5.680 mulheres e o câncer de endométrio, 5.900 mulheres. Em conjunto, representam quase $25 \%$ dos tumores em mulheres, excluindo tumores de pele não melanomas ${ }^{1}$. Cerca de 5 a $10 \%$ desses tumores podem ser hereditários.

Em que situações a história familiar de uma mulher em seguimento regular ginecológico levanta a suspeita de uma síndrome de câncer hereditário, que deveria suscitar uma investigação especializada? A maior parte dos casos seguidos em ambulatórios de aconselhamento genético de câncer no país são de pacientes com história pessoal de câncer, com ou sem história familiar. O acesso de pessoas sem câncer, mas sob risco de desenvolvê-lo por serem de famílias com síndromes de câncer hereditário, é um dos desafios a serem vencidos para uma prevenção mais eficiente nos casos hereditários.

A importância da detecção dos casos de tumores hereditários se dá em função da possibilidade de recorrência na família, disponibilidade de estratégias específicas de seguimento e prevenção e, em algumas situações, abordagens terapêuticas eficientes. $\mathrm{O}$ grande desafio é a detecção, dentre todos os casos de câncer, dos que caracterizariam síndromes de câncer hereditário, necessitando de atenção diferenciada em relação aos casos esporádicos.

Entre as ferramentas mais importantes disponíveis para identificação de mulheres ou familiares com risco de serem acometidos por síndromes de predisposição a câncer estão a história pessoal e familiar de câncer. Algumas informações podem sugerir que a paciente com história pessoal de câncer possa ter uma síndrome de predisposição hereditária a câncer (Quadro 1) $)^{2}$, com ou sem história familiar associada. Especificamente para mama, ovário e endométrio algumas dessas características devem ser cuidadosamente investigadas. A idade de aparecimento do primeiro tumor, por exemplo, deve ser considerada relevante se ocorrer antes dos 50 anos para câncer de mama e endométrio, mas é indiferente para indicação de

Correspondência

Victor Evangelista de Faria Ferraz Departamento de Genética da Faculdade de Medicina de Ribeirãa Preto da Universidade de São Paulo Avenida Bandeirantes, 3.900 - Monte Alegre CEP: $14049-900$

Ribeirõo Preto (SP), Brasi

Recebido

$11 / 03 / 2014$

Aceito com modificacões

$01 / 08 / 2014$
'Departamento de Genética, Faculdade de Medicina de Ribeirão Preto, Universidade de São Paulo - USP - Ribeirão Preto (SP), Brasil. 2Instituto de Biologia, Universidade Estadual de Campinas - UNICAMP - Campinas (SP), Brasil. Conflito de interesses: não há 
Quadro 1. Dados relevantes para suspeita de síndromes de câncer hereditário levando em consideração a história pessoal de câncer

Dados da história pessoal

Tumores primários múltiplos (no mesmo órgão ou em órgãos diferentes)

Tumores bilaterais em órgãos pares

Tumores multifocais

Diagnóstico de câncer em idade mais precoce do que a esperada para o tumor

Tipo histológico raro

Tumor em indivíduo de sexo normalmente não afetado

Tumor associado com defeitos congênitos

Tumor associado com lesões precursoras herdadas

Tumores associados a lesões cutâneas reconhecidas como relacionadas às síndromes

Fonte: adaptado de Lindor et al. ${ }^{2}$

investigação em câncer epitelial de ovário. Outra peça de investigação importante é a possibilidade de investigação imuno-histoquímica nos tumores. Para câncer de mama, por exemplo, a imuno-histoquímica mostrando ausência de receptores de estrógeno, progesterona e HER2/neu é indicação suficiente para investigação de mutações em BRCA1 e BRCA2 em qualquer idade. Para os cânceres de endométrio, atualmente, preconiza-se a investigação por imuno-histoquimica das proteínas associadas ao sistema de reparo de malpareamento, cujo comprometimento pode estar associado à síndrome de Lynch $^{3,4}$. A história familiar de câncer é um importante auxiliar para investigação das síndromes de predisposição ao câncer, associada à história pessoal (Quadro 2) ${ }^{2}$. Recentemente, foram publicadas recomendações específicas para a abordagem da história de câncer na família pelos oncologistas, que certamente podem ser estendidas a outras especialidades (Quadro 3$)^{5}$. São recomendações que permitem uma seleção mais apurada dos casos que eventualmente devem ser encaminhados para investigação especializada, seguimento específico e aconselhamento genético.

Sucintamente, sem o objetivo de abranger todas as síndromes hereditárias que envolvem mama, ovário e endométrio, destacam-se aqui algumas das neoplasias mais relevantes e o papel da história familiar: a síndrome de câncer de mama e ovário hereditários, síndrome de Li-Fraumeni, síndrome de Cowden e a síndrome de Lynch. O Quadro 4 mostra os cânceres do escopo deste texto associados a cada uma delas.

\section{Síndrome de câncer de mama e ovário hereditários (mama e ovário)}

Cerca de $5-10 \%$ dos tumores de mama são hereditários ${ }^{6}$, e a síndrome mais prevalente que pode cursar com câncer de mama é a síndrome de câncer de mama e ovário hereditários (HBOC), respondendo por cerca de 60 a $80 \%$ dos casos. A síndrome é causada por mutações patogênicas nos genes BRCA1 e BRCA2 que, quando presentes, conferem um risco cumulativo de $80-85 \%$
Quadro 2. Dados relevantes para suspeita de sindromes de câncer hereditário levando em consideração a história familiar de câncer

Dados da história familiar

Dois ou mais familiares de primeiro grau com tumores da mesma localização ou tumores relacionados

Dois ou mais familiares de primeiro grau com tumores raros

Dois familiares em duas gerações com tumores da mesma localização ou tumores relacionados

Evidência de transmissão autossômica dominante

Constelação de tumores característicos de uma síndrome específica

Fonte: adaptado de Lindor et al. ${ }^{2}$

Quadro 3. Elementos necessários para uma história familiar de câncer minimamente adequada

Importante na história familiar

Avaliação de parentes de $1^{0}$ grau: pais, filhos e irmãos

Avaliação de parentes de $2^{0}$ grau: tios e tias, avós, netos e netas, sobrinhos e sobrinhas, meio-irmão e meia-irmã

Avaliar sempre tanto o lado paterno como o materno

Indicar a etnia

Idade de diagnóstico de cada caso

Tipo do câncer primário de cada caso

Resultados de eventuais testes moleculares realizados

Quadro 4. Síndromes de câncer hereditário mais comuns associadas tipicamente aos carcinomas de mama, ovário e endométrio

\begin{tabular}{|c|c|c|c|c|}
\hline Sindrome & $\begin{array}{c}\text { Genes } \\
\text { envolvidos }\end{array}$ & $\begin{array}{l}\text { Câncer de } \\
\text { mama }\end{array}$ & $\begin{array}{l}\text { Câncer de } \\
\text { ovário }\end{array}$ & $\begin{array}{l}\text { Câncer de } \\
\text { endométrio }\end{array}$ \\
\hline $\begin{array}{l}\text { Síndrome de câncer } \\
\text { de mama e ovário } \\
\text { hereditários }\end{array}$ & BRCA1 e BRCA2 & + & + & - \\
\hline $\begin{array}{l}\text { Sindrome de } \\
\text { Cowden }\end{array}$ & PTEN & + & - & + \\
\hline $\begin{array}{l}\text { Sindrome de Li- } \\
\text { Fraumeni }\end{array}$ & TP53 & + & $\cdot$ & - \\
\hline Sindrome de Lynch & $\begin{array}{l}\text { MLH1, MSH2, } \\
\text { MSH6 e PMS2/ } \\
\text { EPCAM }\end{array}$ & - & + & + \\
\hline
\end{tabular}

de desenvolver câncer de mama durante toda vida e até $50 \%$ de risco para o câncer de ovário.

Segundo o National Comprehensive Cancer Network $(\mathrm{NCCN})^{3}$, há indicação para o teste genético relacionado a HBOC quando o indivíduo preenche os seguintes critérios:

1. indivíduos de família com histórico de mutação nos genes BRCA1 e/ou BRCA2;

2. história pessoal de câncer de mama +1 ou mais características a seguir:

- idade do diagnóstico $\leq 45$ anos;

- $\quad$ idade do diagnóstico $\leq 50$ anos e/ou $\geq 1$ parente próximo ( $1^{\circ}, 2^{\circ}$ e $3^{\circ}$ graus) com câncer de mama em qualquer idade e/ou uma história familiar limitada ou desconhecida;

- idade do diagnóstico $\leq 60$ anos, com câncer de mama triplo negativo;

- diagnóstico em qualquer idade, com $\geq 1$ parente próximo com câncer de mama $\leq 50$ anos e/ou $\geq 2$ 
parentes próximos $\left(1^{\circ}, 2^{\circ}\right.$ e $3^{\circ}$ graus) com câncer de mama em qualquer idade e/ou $\geq 1$ parente próximo $\left(1^{\circ}, 2^{\circ}\right.$ e $3^{\circ}$ graus) com câncer epitelial de ovário e/ou $\geq 2$ parentes próximos $\left(1^{\circ}, 2^{\circ} \mathrm{e}\right.$ $3^{\circ}$ graus) com câncer pancreático ou câncer de próstata (Gleason 27 ) em qualquer idade e/ou um parente próximo $\left(1^{\circ}, 2^{\circ}\right.$ e $3^{\circ}$ graus $)$ do sexo masculino com câncer de mama e/ou um indivíduo de etnias de alto risco (exemplo: Judeus Ashkenazi).

história pessoal de câncer epitelial de ovário; história pessoal de câncer de mama em homens; história pessoal de câncer pancreático ou câncer de prostata (Gleason $\geq 7$ ) em qualquer idade, com $\geq 2$ parentes próximos $\left(1^{\circ}, 2^{\circ}\right.$ e $3^{\circ}$ graus) com câncer de mama e/ou câncer de ovário e/ou câncer pancreático e/ ou câncer de prostata (Gleason 27 ) em qualquer idade; - indivíduo com história pessoal de câncer pancreático em qualquer idade e ancestralidade Judeus Ashkenazi, com $\geq 1$ parente próximo $\left(1^{\circ}, 2^{\circ}\right.$ e $3^{\circ}$ graus) com câncer de mama e/ou ovário e/ou câncer pancreático e/ou câncer de próstata (Gleason $\geq 7$ ) em qualquer idade.

6. indivíduos apenas com história familiar (a interpretação dos resultados do teste genético de indivíduos sem história pessoal de câncer deve ser discutida):

- algum parente próximo ( $1^{\circ}$ e $2^{\circ}$ graus) da família preenchendo um dos critérios anteriores;

- parente em $3^{\circ}$ grau com câncer de mama e/ou câncer de ovário, com $\geq 2$ parentes próximos $\left(1^{\circ}, 2^{\circ}\right.$ e $3^{\circ}$ graus) com câncer de mama (no mínimo um com diagnóstico $\leq 50$ anos ) e/ou câncer de ovário.

Quanto ao câncer de ovário, Ledermann et al. ${ }^{7}$ publicaram recomendações adotadas pela European Society of Medical Oncology, reforçando o papel da história familiar na investigação causal. Referem que, apesar de apenas cerca de $10 \%$ dos tumores estarem ligados a mutações de BRCA1 e BRCA2, alterações nesses genes conferem risco de desenvolvimento de câncer de ovário em 15 a $45 \%$ ao longo da vida.

$\mathrm{O}$ American College of Obstetricians and Gynecologists ${ }^{8}$ recomenda que mulheres com mutações em BRCA1/2 ou com história familiar suspeita devam ser encaminhadas para aconselhamento genético formal para avaliar melhor o risco de câncer, incluindo o risco de câncer de ovário. A triagem com dosagens séricas de CA 125 e ultrassonografia transvaginal a cada 6 meses tem sido recomendadas para mulheres de alto risco pela National Comprehensive Cancer Network ${ }^{3}$. O American College of Obstetricians and Gynecologists ${ }^{8}$ também recomenda que a salpingooforectomia de redução de risco, que inclui a remoção dos ovários e das trompas de Falópio, na sua totalidade, deva ser oferecida para mulheres com mais de 40 anos com mutações BRCA1 ou BRCA2.

\section{Síndrome de Li-Fraumeni (mama)}

A síndrome de Li-Fraumeni (SLF) é uma síndrome hereditária de predisposição ao câncer, de alta penetrância, na qual portadores de mutações patogênicas no gene TP53 apresentam um risco cumulativo de até $90 \%$ para o desenvolvimento de um amplo espectro de cânceres diagnosticados geralmente antes dos 45 anos. Os tipos de cânceres mais frequentes incluem sarcoma, câncer da mama, tumores cerebrais e carcinomas adrenocorticais. Entretanto, outros cânceres também são observados em uma menor frequência, tais como linfomas, câncer gástrico e melanoma ${ }^{9-11}$. Famílias que não apresentam o fenótipo clássico da síndrome são denominadas Li-Fraumeni like (LFL) ou Li-Fraumeni variante ${ }^{12,13}$.

Segundo o National Comprehensive Cancer Network ${ }^{3}$, há indicação para o teste genético relacionado a SLF quando o indivíduo preenche os seguintes critérios:

1. indivíduos de uma família com uma mutação conhecida no gene TP53;

2. critério da SLF clássica:

- combinação de um indivíduo com sarcoma diagnosticado com $<45$ anos de idade e um parente de primeiro grau com câncer diagnosticado com $<45$ anos de idade e um parente de primeiro ou segundo grau com câncer diagnosticado com $<45$ anos ou com sarcoma em qualquer idade. critérios Chompret:

- indivíduo com tumor do spectrum da SLF (exemplos: sarcoma, osteosarcoma, tumor cerebral, câncer de mama, carcinoma adrenocortical, leucemia e câncer de pulmão bronquioalveolar) diagnosticado antes dos 36 anos de idade e no mínimo um parente de primeiro ou segundo grau com qualquer um dos cânceres mencionados (outro que não câncer de mama, se o probando tiver câncer de mama) diagnosticados antes dos 56 anos ou com múltiplos cânceres primários em qualquer idade;

- indivíduo com tumores primários múltiplos (exceto múltiplos tumores de mama), sendo 2 deles pertencentes ao espectro da SLF, diagnosticado antes dos 46 anos de idade;

- indivíduo com carcinoma adrenocortical ou carcinoma plexus choroid em qualquer idade, independentemente da história familiar;

4. câncer de mama precoce: indivíduo com câncer de mama diagnosticado com $<35$ anos de idade pode fazer o pedido do teste genético do gene TP53 simultaneamente ao dos genes BRCA1 e BRCA2 ou após um teste genético negativo para BRCA1/BRCA2. 
Especialmente no sul do Brasil, a mutação TP53 R337H, localizada no éxon 10 do gene TP53, tem sido associada ao aumento do risco de desenvolvimento do câncer de mama em mulheres ${ }^{14-16}$. Essa mutação é responsável pela troca de uma arginina por uma histidina (CGC para CAC) no códon 337 e foi primeiramente associada com tumores adrenocorticais em crianças $^{17}$.

Achatz et al. realizaram um screening da mutação TP53 p.R337H em 45 indivíduos de famílias não relacionadas com histórico de câncer sugestivo para SLF. Seis desses casos (13\%) eram portadores da mutação, sendo que o tipo de tumor mais comum nessas famílias era o câncer de mama $(30,4 \%)^{14}$.

A patogenicidade da mutação $\mathrm{R} 337 \mathrm{H}$ é questionável devido ao baixo número de estudos funcionais realizados até o momento. Entretanto, como a mutação TP53 p. $\mathrm{R} 337 \mathrm{H}$ apresenta uma alta frequência em mulheres brasileiras com câncer de mama ${ }^{14-16,18}$, o teste genético para essa mutação deveria ser realizado em todas as pacientes brasileiras com câncer de mama, independentemente da idade do diagnóstico, com história familiar contendo o espectro de tumores da SLF/LFL.

\section{Síndrome de Cowden (mama, endométrio)}

A síndrome de Cowden (CS) é uma genodermatose autossômica dominante associada a mutações no gene PTEN em cerca de $80 \%$ dos casos. Caracteriza-se pela presença de lesões mucocutâneas consideradas patognomônicas (triquilemomas, papilomatose mucocutânea, ceratose acral) e presentes em mais de $90 \%$ dos casos até a $3^{\text {a }}$ década de vida, associadas a macrocefalia e a presença de tumores de mama, tiroide e endométrio, considerados critérios maiores. São considerados critérios menores para o diagnóstico outras lesões de tiroide, deficiência intelectual, hamartomas de trato digestivo, lipomas, doença fibrocística da mama, fibroides uterinos, fibromas e tumores ou anomalias genitourinários. A suspeita se dá pela presença de um achado patognomônico, dois ou mais critérios maiores, um critério maior associado a três menores, ou quatro critérios menores. História familiar, associada a um achado patognomônico ou um critério maior, ou ainda dois critérios menores são suficientes para iniciar-se a investigação ${ }^{19}$. O risco de desenvolvimento de câncer de mama, em geral carcinoma ductal invasivo, em pacientes com síndrome de Cowden é de cerca de 50\% durante a vida. O risco para desenvolvimento de câncer de endométrio é de cerca de 13 a $19 \%{ }^{19}$.

\section{Síndrome de Lynch (endométrio, ovário)}

Entre 2 e $5 \%$ dos cânceres de endométrio podem ser ligados a mutações germinativas de genes relacionados ao sistema de reparo de malpareamento, caracterizando uma síndrome de câncer hereditário conhecida por Sindrome de Lynch. A síndrome de Lynch envolve a predisposição autossômica dominante a câncer colorretal, câncer de endométrio e outros. Está ligada a mutações nos genes MLH1, MSH2, MSH6 e PMS2, pertencentes ao sistema de reparo de malpareamento de DNA, genes cuja expressão pode ser verificada por imuno-histoquímica no tumor. Em alguns casos, está ligado a deleções germinativas no gene EPCAM, que indiretamente silenciam o gene $\mathrm{MSH} 2^{20}$. Uma forma mais genérica de verificar se o sistema de reparo em questão está afetado é através do exame de instabilidade de microssatélites por PCR no tumor, que não aponta especificamente qual proteína está alterada ou ausente ${ }^{2,21}$.

Mulheres com síndrome de Lynch têm risco de 30 a $70 \%$ de apresentar câncer de endométrio durante a vida. Há dúvidas sobre a estratégia mais custo-eficaz para detecção desses casos, havendo propostas de avaliação do sistema de reparo de malpareamento no tumor, por imuno-histoquímica ou instabilidade de microssatélites nos tumores de pacientes com menos de 50 anos, com menos de 60 anos de idade em determinadas condições ligadas a características histopatológicas, ou mesmo universalmente ${ }^{22}$. Mesmo assim, fica claro que, para mulheres com carcinoma de endométrio em idade inferior a 50 anos, essa avaliação deveria ser rotineira.

\section{Diagnóstico, seguimento e tratamento}

A National Compreehensive Cancer Network ${ }^{3}$ mantém atualizados protocolos consensuais para os casos de investigação de casos de alto risco, assim como o seguimento de casos de pacientes com síndromes de câncer hereditário, ou sob suspeita, que envolvem cânceres de mama, ovário e endométrio. Esses protocolos podem ser acessados em http://www.nccn.org/professionals/physician_gls/f_guidelines.asp\#detection e permitem uma orientação mais específica, voltada para a suspeita diagnóstica.

\section{Aconselhamento genético}

O aconselhamento genético para pacientes e familiares com ou sob risco de síndromes de câncer hereditário é essencial para a abordagem adequada a essa problemática. $\mathrm{O}$ sequenciamento dos genes das síndromes suspeitadas deve sempre ser acompanhado de aconselhamento pré e pós-teste, explicações sobre as possibilidades e limitações dos exames a serem realizados, as possibilidades de prevenção e a apresentação da estratégia de seguimento ${ }^{3}$.

A melhoria da qualidade da obtenção da história familiar de câncer em mulheres em seguimento ginecológico regular é uma necessidade para uma detecção mais precoce dos casos que merecem uma estratégia de prevenção diferenciada. Certamente, um desafio que os ginecologistas podem enfrentar com facilidade. 
1. Brasil. Ministério da Saúde. Instituto Nacional de Câncer José Alencar Gomes da Silva. Coordenação de Prevenção e Vigilância. Estimativa 2014: incidência de câncer no Brasil. Rio de Janeiro: INCA; 2014

2. Lindor NM, McMaster ML, Lindor CJ, Greene MH; National Cancer Institute, Division of Cancer Prevention, Community Oncology and Prevention Trials Research Group. Concise handbook of familial cancer susceptibility syndromes-second edition. J Natl Cancer Inst Monogr. 2008;(38): 1-93.

3. National Comprehensive Cancer Network [Internet]. Genetic/ familial high-risk assessment: breast and ovarian: V.1.2014. Fort Washington: NCCN; 2014 [cited 2014 Jul 12]. (NCCN Clinical Practice Guidelines in Oncology). Available from: <http:// demystifyingmedicine.od.nih.gov/DM10/0413-BreastCancer/ NCCN\%20br\%20genetics_screening.pdf>

4. Egoavil C, Alenda C, Castillejo A, Paya A, Peiro G, Sánchez-Heras $A B$, et al. Prevalence of Lynch syndrome among patients with newly diagnosed endometrial cancers. PLoS One. 2013;8(1 1):e79737.

5. American Society of Clinical Oncology [Internet]. ASCO issues new recommendations for family history taking in oncology setting. Alexandria: ASCO; 2014 [cited 2014 Feb 20]. Available from: <http://www.asco.org/advocacy/asco-issues-new-recommendationsfamily-history-taking-oncology-setting>

6. Chacón RD, Costanzo MV. Triple-negative breast cancer. Breast Cancer Res. 2010;12 Suppl 2:S3.

7. Ledermann JA, Raja FA, Fotopoulou C, Gonzalez-Martin A, Colombo N, Sessa C, et al. Newly diagnosed and relapsed epithelial ovarian carcinoma: ESMO Clinical Practice Guidelines for diagnosis, treatment and follow-up. Ann Oncol. 2013;24 Suppl 6:vi24-32.

8. American College of Obstetricians and Gynecologists Committee on Gynecologic Practice. Committee Opinion No. 477: the role of the obstetrician-gynecologist in the early detection of epithelial ovarian cancer. Obstet Gynecol. 2011 ; 1 17(3):742-6.

9. Li FP, Fraumeni JF Jr. Sofft-tissue sarcomas, breast cancer, and other neoplasms. A familial syndrome? Ann Intern Med. 1969;71 (4):747-52.

10. Nichols KE, Malkin D, Garber JE, Fraumeni JF Jr, Li FP. Germline p53 mutations predispose to a wide spectrum of early-onset cancers. Cancer Epidemiol Biomarkers Prev. 2001;10(2):83-7.
11. Birch JM, Hartley AL, Tricker KJ, Prosser J, Condie A, Kelsey $\mathrm{AM}$, et al. Prevalence and diversity of constitutional mutations in the p53 gene among 21 Li-Fraumeni families. Cancer Res. 1994; 54(5): 1298-304.

12. Eeles RA. Germline mutations in the TP53 gene. Cancer Surv. $1995 ; 25: 101-24$.

13. Chompret A, Brugières L, Ronsin M, Gardes M, Dessarps-Freichey F, Abel A, et al. P53 germline mutations in childhood cancers and cancer risk for carrier individuals. Br J Cancer. 2000;82(12): 1932-7.

14. Achatz MI, Olivier M, Le Calvez F, Martel-Planche G, Lopes A, Rossi BM, et al. The TP53 mutation, $\mathrm{R} 337 \mathrm{H}$, is associated with Li-Fraumeni and Li-Fraumeni-like syndromes in Brazilian families. Cancer Lett. 2007;245(1-2):96-102.

15. Assumpção JG, Seidinger AL, Mastellaro M, Ribeiro RC, Zambetti GP, Ganti R, et al. Association of the germline TP53 R337H mutation with breast cancer in southern Brazil. BMC Cancer. 2008;8:357.

16. Garritano S, Gemignani F, Palmero El, Olivier M, Martel-Planche G, Le Calvez-Kelm F, et al. Detailed haplotype analysis at the TP53 locus in p.R337H mutation carriers in the population of Southern Brazil: evidence for a founder effect. Hum Mutat. 2010;31 (2):143-50.

17. Figueiredo BC, Sandrini R, Zambetti GP, Pereira RM, Cheng C, Liu $W$, et al. Penetrance of adrenocortical tumours associated with the germline TP53 R337H mutation. J Med Genet. 2006;43(1):91-6.

18. Cury NM, Ferraz VE, Silva WA Jr. TP53 p.R337H prevalence in a series of Brazilian hereditary breast cancer families. Hered Cancer Clin Pract. 2014;12(1):8.

19. Blumenthal GM, Dennis PA. PTEN hamartoma tumor syndromes. Eur J Hum Genet. 2008; 16(1 1):1289-300.

20. Kempers M, Kuiper RP, Ockeloen CW, Chappuis PO, Hutter P, Rahner $N$, et al. Risk of colorectal and endometrial cancers in EPCAM deletion-positive Lynch syndrome: a cohort study. Lancet Oncol. $2011 ; 12(1): 49-55$.

21. Goodenberger $M$, Lindor NM. Lynch syndrome and MYH-associated polyposis: review and testing strategy. J Clin Gastroenterol. $2011 ; 45(6): 488-500$.

22. Moline J, Mahdi H, Yang B, Biscotti C, Roma AA, Heald B, et al. Implementation of tumor testing for Lynch syndrome in endometrial cancers at a large academic medical center. Gynecol Oncol. 2013;130(1):121-6. 